Original language English

Journal MRS Online Proceedings Library

Journal publication date 2011

Volume $\quad 1290$

DOIs

http://dx.doi.org/10.1557/opl.2011.525

State Published

\title{
Excited Multiplets of Eu in GaN
}

Ben Hourahine

Department of Physics, SUPA, 107 Rottenrow, University of Strathclyde, Glasgow, Scotland, GL4 0NG, UK

\begin{abstract}
A method to calculate the multiplet states of lanthanide impurities in solids is presented. This approach is based on a semi-empirical density functional method which includes corrections to account for the correlation and spin-orbit coupling of the $4 f$ electrons. Specific multiplet states of the rare earth are produced by constraining the system. This approach is then used to investigate some of the properties of substitutional europium impurities in gallium nitride, reproducing the relative energy of two multiplets, and discussing a potential excitation mechanism for these centers.
\end{abstract}

\section{INTRODUCTION}

The optical spectra of trivalent rare-earth impurities in semiconductors are dominated by inter- $f$-shell transitions between multiplets of the ions, which themselves are labelled according to the atomic Russel-Saunder scheme [1]. These states are challenging to model theoretically for several reasons. In the general case of lanthanides with partly filled $4 f$ shells, the typical choice of mean-field density functional theory (DFT) produces a qualitatively incorrect description of the levels. The states are also split by spin-orbit coupling, hence methods beyond the usual scalar relativistic correction of the energy levels of heavy atoms are also needed. Finally, conventional DFT is a ground state technique and while time-dependent DFT does allow access to excitation energies, the currently available functionals perform poorly for both ionic systems and charge transfer excitations.

The experimental excitation processes of rare-earths are also contentious, with models based on either universal alignment of atomic energy levels to the semiconductor band-structure [2], or structured isoelectronic impurities (dopants which behave sufficiently similarly to a constituent element of the crystal, but posses further internal structure)[3].

The semi-empirical density-functional based tight binding (DFTB) method [4,5] has been extended to treat the gross physical effects present for lanthanide impurities in solids. The electronic properties of Eu have previously been investigated using DFTB[6], using a groundstate non-relativistic form of the current method. In this study, Sanna et al. found an acceptor 
level for europium, where the total number of $4 \mathrm{f}$ electrons changes from 6 (trivalent) to 7 (divalent) at $\mathrm{Ev}+2.70 \mathrm{eV}$, decreasing to $\mathrm{Ev}+1.58 \mathrm{eV}$ if the negative charge state is structurally relaxed. This type of europium acceptor level has also been suggested on the basis of selfinteraction corrected DFT[7] in cubic GaN.

A short description of the DFTB method and its extensions to treat $4 f$ electrons are given, followed by a discussion of the ${ }^{7} \mathrm{~F}_{0}$ ground and ${ }^{5} \mathrm{D}_{0}$ excited states of subsitutional trivalent europium in GaN.

\section{THEORY}

The DFTB[4] method is a form of non-orthogonal two center tight-binding where the onsite and hopping integrals are explicitly calculated from full DFT. A minimal basis appropriate for the condensed matter environment is constructed by confining atomic states in a quadratic potential. Similarly the total energy in DFTB includes fitted pairwise repulsive interactions between atoms, again obtained from DFT. The effects of charge transfer are also incorporated through self-consistent inclusion of damped electrostatic interactions between net charges for atomic shells (using Mulliken populations). Similarly, spin-polarization is included through a Stoner-like interaction between magnetic moments[8]. This type of model can readily be extended to allow for non-collinear spin patterns[9], by using a two-component formalism for the single particle states, where the direction of magnetization then can vary between different atomic shells and between different atomic sites[8]. This leads to a total energy and hamiltonian of the general form

$$
\begin{gathered}
E=\operatorname{Tr} H^{0} \cdot \rho_{I}+\frac{1}{2} \sum \Delta q_{\mu}(\rho) \cdot W_{\mu v} \cdot \Delta q_{v}(\rho)+\sum_{a b} E_{r e p .}\left(\left|r_{a}-r_{b}\right|\right) \\
H_{i j}=H_{i j}^{0}+\frac{1}{2} \sum_{k}\left(S_{i k} W_{k l} \Delta q_{l}(\rho)+S_{j k} W_{k l} \Delta q_{l}(\rho)\right)
\end{gathered}
$$

The first term in the energy is the trace of the product of only the $\sigma_{\mathrm{I}}$ Pauli matrix projected part of density matrix $(\rho)$ and the non-self-consistent hamiltonian $H^{0}$. The $\Delta q_{\mu}$ terms are fluctuation of the charge and magnetization Mulliken populations from the neutral spin-unpolarized reference state of the system for atomic shell $\mu$. The coupling between these fluctations, $W$, is on-site in the case of magnetic interactions, but contains both long range coulombic and short range exchangecorrelation contributions for charge fluctuations $[4,8,10]$. The final term in the total energy is the short ranged pairwise repulsive interaction between the atoms in the system (containing most of the DFT double counting contribution for the reference system). These expressions can then be augmented with corrections for the strong correlation between the $4 f$ electrons. In this work the atomic-like $\mathrm{LDA}+\mathrm{U}$ correction[11] is included, this contribution pushes the on-site electronic states towards being either fully localized on the rare earth or fully empty. In this material system, this term acts to move state which would otherwise be partially filled rare-earth levels from the GaN band gap[10,12]. Following the discussion in Ref. [13] the Hubbard- $U$ values used for the $\mathrm{Eu} 5 d$ and $4 f$ shells are half of the isolated atomic values.

Atomic Russel-Saunders coupling can be included in the two-component DFTB hamiltonian [8], using a spin-block structure of the form

$$
H_{L S}=\frac{\xi}{2}\left(\begin{array}{cc}
L_{z} & L^{-} \\
L^{+} & -L_{z}
\end{array}\right)
$$


Where the angular momentum operators are used on the Mulliken projected non-collinear density matrix[14]. Values of $\xi$ for $\mathrm{GaN}$ and the $\mathrm{Eu} 4 f$ shell were taken from the literature $[15,16]$.

\section{$\underline{\text { Excited states and constraints }}$}

While conventional DFT (and DFTB by virtue of its DFT origins) is a ground state theory, it has been established that the lowest energy state with a specific symmetry (which may itself be an excited state of the full system) is actually rigorously accessible within this theory [17]. Following a somewhat similar track, constraining the system such that a state with specific properties[18] becomes the ground state (by finding a $v$ - or $v$-ensemble ground state in a constructed external potential), either by using undetermined multipliers[19], or by adding a penalty functional [14] is also possible in DFT. In the present work, a quadratic constraining potential is used (where neccesary) to force the ground state to posses a particular total magnetic or orbital moment. The free energy of the system in such a potential is of the form

$$
F=E+V\left(P[\rho]-P^{0}\right)^{\mathrm{k}}
$$

Where $\mathrm{P}$ is the functional to determine the particular property to constrain. For the present application, $\kappa=2$, leading to a quadratic penalty for the ground state, in this case the strength of the constraint is proportional to $\mathrm{V}$, which is taken to be 1 Hartree for the purposes of the current study. This energy expression then also leads to an associated contribution to the hamiltonian[14]. The internal energy, E, of the system in this external field is the term relevant to the present discussion. The constraints used here are the magnitudes of the spin and/or angular momentum of the Mulliken-projected density matrix.

\section{RESULTS}

The DFTB parameterization from Refs. [6,10,20] have been used with a $4 \times 4 \times 3$ wurtzite supercell with $4^{3}$ Monkhorst-Pack $k$-points [21] and a Fermi distribution temperature of $300 \mathrm{~K}$. $\mathrm{Eu}$ is placed on a Ga site, and the geometry and lattice constants fully relaxed using collinear spin-polarized DFTB. This structure is then used for the subsequent calculations. In the resulting non-collinear calculations, the $4 f$ magnetic moment is found to align with the wurtzite c-direction (though, as with $\mathrm{Gd}[14]$, the magneto-crystalline anisotropy is small). 

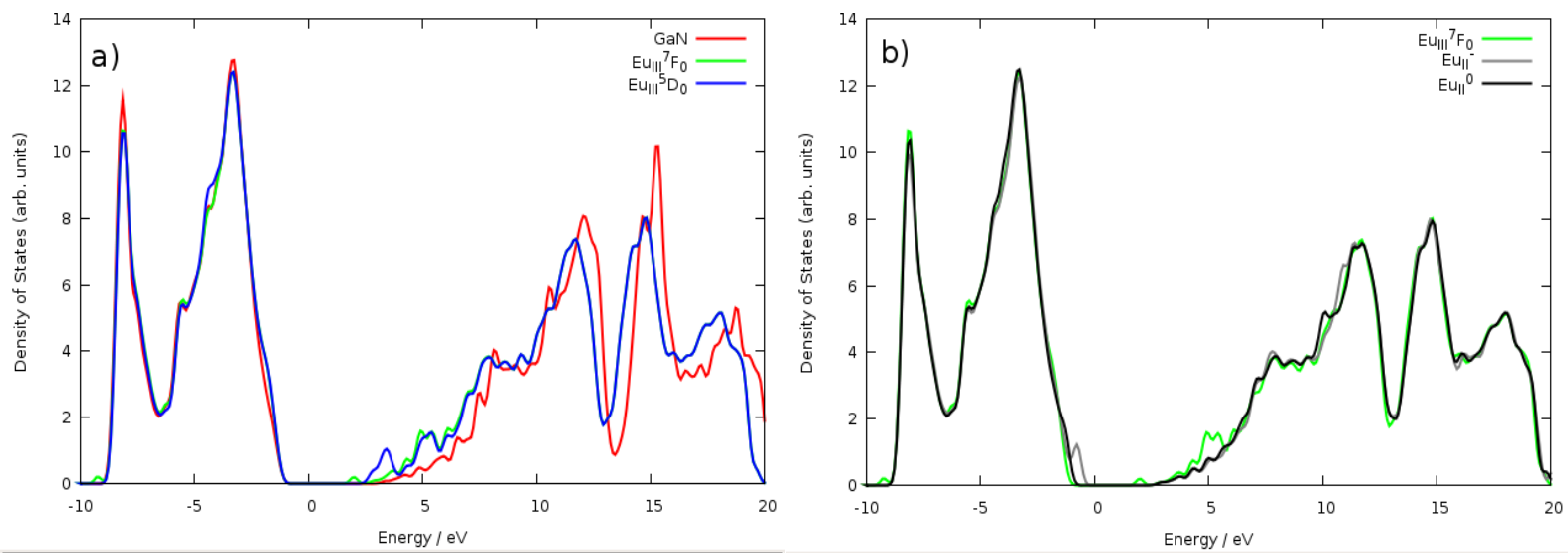

Figure 1. Density of states for subsitutional Eu in $\mathrm{GaN}$ a) DOS shown for bulk $\mathrm{GaN}$ and EuIII in ground and excited states, b) EuIII compared with the filled and empty EuII acceptor state. The energy scale is aligned to place the Fermi energy at $0 \mathrm{eV}$, and does not account for the average potential of the cell.

The density of states of the $\mathrm{GaN}$ host and the ${ }^{7} \mathrm{~F}_{0}$ ground state multiplet of trivalent Eu is shown in Fig 1a. The limitations of the DFTB minimal basis are apparent in the conduction band density of states, which unlike the well represented valence band, show a distinct shift due to the presence of Eu. The density of states show $f$-related features at the conduction band edge (and at around 4 and $9 \mathrm{eV}$ below the Fermi energy). The DOS for the ${ }^{5} \mathrm{D}_{0}$ state is also shown in figure 1a, this state is produced by constraining the magnitude of the spin and orbital moments, leading to the states shown in Table 1.

\begin{tabular}{|l|l|l|l|l|l|l|l|l|}
\hline & \multicolumn{9}{l|}{$4 f$} & \multicolumn{3}{l|}{$5 d$} \\
\hline & $\mathrm{q} / \mathrm{e}$ & $\mathrm{M}_{\mathrm{S}} / \mu_{\mathrm{B}}$ & $\mathrm{M}_{\mathrm{L}} / \mathrm{g} \mu_{\mathrm{B}}$ & $\mathrm{M}_{\mathrm{J}} / \mathrm{g} \mu_{\mathrm{B}}$ & $\mathrm{q} / \mathrm{e}$ & $\mathrm{M}_{\mathrm{S}} / \mu_{\mathrm{B}}$ & $\mathrm{M}_{\mathrm{L}} / \mathrm{g} \mu_{\mathrm{B}}$ & $\mathrm{M}_{\mathrm{J}} / \mathrm{g} \mu_{\mathrm{B}}$ \\
\hline EuIII $\left({ }^{7} \mathrm{~F}_{0}\right)$ & 6.04 & 5.94 & -2.95 & 0.02 & 1.24 & 0.10 & 0.00 & 0.05 \\
\hline EuIII $\left({ }^{5} \mathrm{D}_{0}\right)$ & 6.06 & 4.08 & -2.00 & 0.04 & 1.23 & 0.07 & 0.00 & 0.04 \\
\hline Eu'II & 6.98 & 6.93 & 0.01 & 3.47 & 1.05 & 0.07 & 0.00 & 0.03 \\
\hline Eu $^{0} \mathrm{II}$ & 6.97 & 6.92 & 0.02 & 3.46 & 0.71 & 0.01 & 0.00 & 0.00 \\
\hline
\end{tabular}

Table 1. The ground state and ${ }^{5} D_{0}$ multiplets, showing the number of electrons, magnetization, orbital momentum and total angular momentum for the $f$ and $d$ shell. The divalent states of Eu are also shown.

The resulting states are reasonable matches to the two $\mathrm{J}=0$ states considered. The ${ }^{5} \mathrm{D}_{0}$ state is 2.14 $\mathrm{eV}$ higher in energy than the ${ }^{7} \mathrm{~F}_{0}$ ground state, agreeing well with the experimental excitation energy of $2.17 \mathrm{eV}[22]$.

Fig. $1 \mathrm{~b}$ shows the density of states for the filled acceptor state of europium, Eu'II, which when compared with the ${ }^{7} \mathrm{~F}_{0}$ ground state, shows an extra feature just above the valence band maximum and a corresponding loss of the near conduction-band $4 f$ feature. This state is constructed by charging the supercell, however an alternative is to constrain the $4 f$ shell of a neutral system to contain 7 electrons, leading to a state similar to a core exciton, where a hole is bound to a divalent $\mathrm{Eu}$ atom. This excited state is $0.50 \mathrm{eV}$ higher in energy than the ${ }^{7} \mathrm{~F}_{0}$ ground state. 

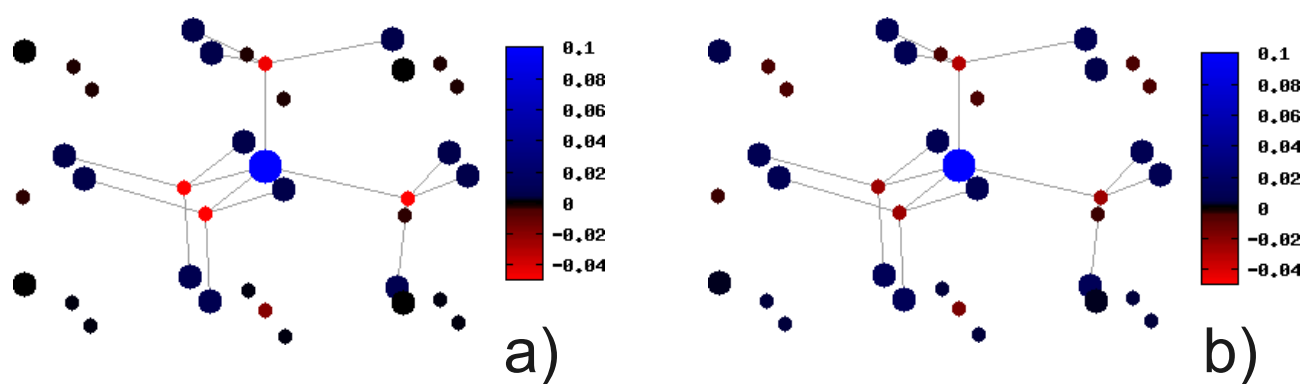

Figure 2. Charge differences surrounding a) Eu-II and b) Eu II compared with the EuIII ${ }^{7} \mathrm{~F}_{0}$ state.

As shown in figure 2, for both the $\mathrm{Eu}^{0 /} \mathrm{II}$ states, the main local charge transfer compared with the EuIII ground state involves the Eu atom and its 4 nitrogen neighbors with the net charge of the Eu becoming around $0.1 \mathrm{e}$ more positive. It is interesting to note that even in the case of the divalent $\mathrm{Eu}$-II state, the magnetic moment of the system is almost entirely associated with the onsite $4 f$ orbitals of the $\mathrm{Eu}(99.4 \%)$.

\section{DISCUSSION}

The presented methodology seems to reproduce the relative energies of the ${ }^{7} \mathrm{~F}_{0}$ and ${ }^{5} \mathrm{D}_{0}$ states well, presumably in part due to the dominant contribution of the spin-orbit coupling to the relative energy of the two multiplets. Further tests with $\mathrm{J} \neq 0$ multiplets should provide a more stringent criteria to judge its reliability. The density of states for the two systems show a shift in the position of the empty $4 f$ related states in the region around the bottom of the conductionband. This is distinct from the changes which occur on filling the proposed acceptor level of Eu, which remove these features but introduce a new structure just above the valence band. In all cases the angular momentum of the system remains firmly associated with the $4 \mathrm{f}$ states of $\mathrm{Eu}$ impurity.

The acceptor level has not been experimentally observed as far as I am aware. However, the excitonic-like energy to excite Eu can be approximately estimated, by combining the excitation energy from the ground state to the $\mathrm{Eu}^{0} \mathrm{II}$ configuration, with the energy to release a hole to a weakly bound state around the valence band maximum (which can be approximated by the position of the acceptor level for the un-relaxed structure predicted by Sanna et al.). This yields a total energy of $3.20 \mathrm{eV}$, which is in qualitative agreement with the recent PLE measurements of Roqan et al.[23] on high-pressure annealed Eu doped GaN, where they suggest that the non-resonant excitation path for EuIII in GaN requires light of above band-gap energy. It may be possible to obtain a more rigorous estimate of this energy if it is possible to use the Slater-Janak transition state[24] argument between the exciton-like configuration and the excited divalent atomic state.

\section{ACKNOWLEDGMENTS}

The Centre for Complex systems, Strathclyde, are thanked for computational resources. This work has been partly support by the RAINBOW Initial Training Network (PITN-GA-2008213238). 


\section{REFERENCES}

1. O'Donnell and Hourahine, Europ. Phys. J.: Appl. Phys. 3691 (2006).

2. Dorenbos and van der Kolk, Opt. Matt. 301052 (2008).

3. Lozykowski, Phys. Rev. B 4817758 (1993).

4. Frauenheim et al, J. Phys.: Condens. Matter 143015 (2002).

5. Aradi, Hourahine and Frauenheim, J. Phys. Chem. A 111 (2007).

6. Sanna, Frauenheim and Gerstmann, Phys. Rev. B 78085201 (2008).

7. Svane, Christensen, Petit, Szotek and Temmerman, Phys. Rev. B 74165204 (2006).

8. Köhler, Frauenheim, Hourahine, Seifert and Sternberg, J. Phys. Chem A 1115622 (2007).

9. Pickett, J. Korean Phys. Soc.(Proc. Suppl.) 29, S70 (1996).

10. Sanna, Hourahine, Gerstmann, Frauenheim, Phys. Rev. B 76155128 (2007).

11. Anisimov, Aryasetiawan and Lichtenstein, J. Phys. Cond Matt. 9767 (1997).

12. Sanna, Hourahine, Gallauner and Frauenheim, J. Phys. Chem A 111, 5665 (2007).

13. Hourahine et al., J. Phys. Chem A 111, 5671 (2007).

14. Hourahine, Aradi and Frauenheim, J. Phys.: Conf. Ser. 242012005 (2010).

15. Cardona and Christensen, Solid State Comm. 116421 (2000).

16. Carnall, Goodman, Rajnak and Rana, J. Chem. Phys. 90, 3443 (1989).

17. Theophilou, International Journal of Quantum Chemistry 61333 (1997).

18. Dederichs et al., Phys. Rev. Lett. 532512 (1984).

19. Wu and van Voorhis, Phys. Rev. A 7224502 (2005).

20. Simone Sanna, $\mathrm{PhD}$ thesis Universität Paderborn (2003).

21. Pack and Monkhorst, Phys. Rev. B 161748 (1977).

22. G. H. Dieke, "Spectra and energy levels of rare earth ions in crystals", Interscience New York (1968).

23. Roqan et al., Phys. Rev. B 81085209 (2010).

24. Janak, Phys. Rev. B 187165 (1978). 